\title{
Rarefication Effects on Jet Impingement Loads
}

\author{
Shiying Cai ${ }^{1}$, Chunpei Cai ${ }^{2, *}$, Kai Zhang ${ }^{2}$ and Jun $\mathrm{Li}^{3}$ \\ 1 School of Mechanical and Electrical Engineering, Qingdao Science and Technological University, \\ Qingdao 26610, China; NXJ710619@gmail.com \\ 2 Department of Mechanical Engineering-Engineering Mechanics, The Michigan Technological University, \\ Houghton, MI 49931, USA; kazhang@mtu.edu \\ 3 Center for Integrative Petroleum Research, College of Petroleum Engineering \& Geosciences, \\ King Fahd University of Petroleum \& Minerals, Dhahran 31261, Saudi Arabia; junli@kfupm.edu.sa \\ * Correspondence: ccai@mtu.edu; Tel.: +1-906-487-3286
}

Received: 16 August 2017; Accepted: 13 September 2017; Published: 19 September 2017

\begin{abstract}
Rarefication effects on jet impingement loads are studied by comparing recent new formulas at the collisionless flow limit and numerical simulations. The jet exit size is finite, and can be either planar or round. In the simulations, the jets have different degrees of rarefication, with a Knudsen $(K n)$ number ranging from 0 to infinity; i.e., the jet flows can be continuum, collisional, or collisionless. The comparison results indicate that (1) the new surface load formulas are accurate at the collisionless flow limit; (2) in general, the formulas offer upper limits for the peak loads; (3) however, it is improper to assert that local loads always decrease. The new formulas can offer fast estimations of impingement loads. This may be quite helpful for applications in space engineering by significantly reducing the amount of simulations and experiment costs. Those expressions explicitly include non-dimensional parameters, and their contribution and influence on the loads can be studied in a systematic manner (e.g., with a swift parameter study).
\end{abstract}

Keywords: compressible flows; free molecular flow; rarefied gas dynamics; jet impingement; direct simulation Monte Carlo

\section{Introduction}

Rarefied jet and impingement flows are fundamental fluid dynamics problems with many applications. For example, for thin film deposition processes within a vacuum chamber, gaseous jet sprays are critical [1]. Molecule beams [2] are widely used for scientific research studies. Especially, in aerospace and space engineering, dilute jet impingement at a flat plate is usually a practical concern. For example, for station-keeping missions, retro-rockets are commonly adopted, and the related jets may impinge at sensitive surfaces, creating adverse loads which can damage the surfaces, affect satellite control, and jeopardize the missions. For a landing rocket on the Moon surface, the interactions among rocket plumes, lunar dust, and crater development may be a serious issue-especially for multiple-vehicle landing missions [3,4]. For space weather applications [5], plasma jet impingements on spacecrafts (shuttle, station, and satellite) are major concerns. Other applications include thermal/chemical/ion/plasma thruster plumes and jet vacuum pumping by diffusion or ejector pumps [6].

Due to the importance, there are many related numerical simulations, modeling studies, and experimental measurements on jet flows and impingement in the literature. Compared with simulations and experimental measurements, modeling work is faster, less demanding, and parameters are explicitly embedded in the final expressions. In general, analytical models are more rigorous and can lead to superior results compared to empirical models; hence, they are always desired.

In the literature, there are many reports on the development of analytical and explicit formulas for collisionless jet and jet impingement flows, and here we only name a few. For example, Liepmann [7] 
investigated the exact solutions for a collisionless jet out of an orifice. Narasimha derived analytical expressions of the density field for a collisionless jet from a planar or a round exit [8]. The continuum Prandtl-Meyer flow formulas are frequently applied to approximate rarefied jet flow problems $[9,10]$. Simons explained the cosine law model for continuum rocket plume density distributions [11] by using the continuum boundary layer theory and flow entrainment. Numerically, the direct simulation Monte Carlo (DSMC) method [12] is widely adopted to simulate rarefied jet and jet flow problems. Ivanov and his colleagues [13] implemented a special package and used it to study and optimize a Russian space station. Kannenberg and Boyd performed numerical simulations of a rarefied jet impinging at a plate with different inclination angles [14]. Noller [15] proposed the study of a rarefied plume flow with two regions: in the inner core, jet flows can be treated as continuum; many small "starter" surfaces are adopted to discretize the density flowfield wrapping the inner core; the outer regions are treated as free molecular and studied by using the gaskinetic theory and these start surfaces. With this method, Noller successfully computed a rarefied plume density field. Woronowicz [16] performed many numerical calculations of retro-rocket jet impingement on spacecraft surfaces with the collisionless flow approach. Chen [17] used a gaskinetic method to accurately compute collisionless jet flow impingement loads on a solar panel. Dettleef [18] provided a detailed review of work on rarefied jets and jet impingement flows before 1990.

The goal of this paper is to investigate rarefication effects on jet impingement loads at an inclined plate surface. The jet fires from a planar or round nozzle. The simulation results are compared with recently developed jet impingement flow formulas at the collisionless flow limits, and these formulas include several crucial parameters.

The rest of the paper is organized as follows. Section 2 presents the formulas to be tested; Section 3 presents numerical simulation results and discussions; and finally, Section 4 summarizes this study with several conclusions.

\section{New Formulas for Collisionless Compressible Jet Impinging Loads at a Planar Plate}

Even though the past work on rarefied jets and jet impingement flows is significant, there are several unresolved issues, and two of them are listed below.

First, there is not much rigorous development of accurate expressions for rarefied jet and jet impingement flowfield and surface loads. The surface properties are practical for many engineering problems. They include local load distributions (i.e., surface pressure, friction, and heat flux) and the total surface loads, such as total pressure and friction forces, heat flux, and moment over the plate center, and the distance from the impingement force center to the plate center. Obviously, the expressions for "global" properties are more challenging, but they can be evaluated conveniently if the exact expressions for the local load distributions are available.

Another problem is that the past empirical formulas for jet and impingement flows do not include enough physical factors. For example, the concise cosine law [11] formula only includes factors of geometry relations and the reference density at the nozzle exit. The point source flow assumption does not even include factors of the exit geometry, nor exit bulk gas velocity and temperature. There are no formulas for surface loads which include many physical factors (e.g., the surface temperature, surfaces which are specular or diffuse reflective). Here a diffuse reflective surface means when a molecule hits a plate, it bounces back randomly along any direction off the plate; a specular reflective surface means the molecule's velocity component parallel to the surface remains the same, but its normal velocity component is reversed. Empirical and exact analytical expressions for several flowfield and surface properties are not well studied at all; e.g., flowfield velocity and pressure distributions, plate surface forces, and heat loads.

It is well-known that compressible gaseous jet and impingement flows can be classified into four categories, according to different degrees of rarefication or the Knudsen number (Kn) [12], which is defined as the ratio of the molecule mean free path at the nozzle exit to a characteristic length, $K n=\lambda_{0} / H$. The mean free path $\lambda_{0}$ is usually computed with the hard sphere model [12], and $H$ 
is a characteristic length for the flow and it can be the nozzle exit semi-height. These four regimes are: continuum $(K n<0.001)$; velocity-slip and temperature jump $(0.001<K n<0.01)$; transitional $(0.01<K n<1)$; and collisionless (or free molecular, $K n>1)$. Very likely, solutions to jet and jet impingement flows in the velocity-slip and transition regimes can be interpolated from the solutions to the two limits (i.e., continuum and collisionless flow regimes). However, this kind of approach has not investigated because the exact and detailed expressions for the surface loads at the collisionless flow limit have not been available, and especially, the finite size of nozzle exit complicates the surface load expressions significantly.

To adopt an interpolation approach, the following questions shall be answered:

(1) What are the exact jet impingement load solutions at the continuum and collisionless flow limits? For compressible jet and impingement flows from a finite nozzle exit, the corresponding analytical expressions have only recently been studied well. The results include the whole flowfield properties (i.e., density, velocity, pressure, and temperature) and plate surface loads $[19,20]$. The derivation processes are lengthy, and are well explained with two long papers [20]; they are not the focus of this paper, and to keep this paper concise, only the final expressions for the surface loads are summarized in the two following subsections. The challenges of the past investigations were the construction of proper integration domains and the related velocity distribution functions (VDFs) for the jet and the plate surface. Both VDFs are assumed as Maxwellian, and the parameters for the plate are determined by the non-penetration boundary conditions at the plate surface. Gaskinetic theory is used to obtain bulk properties on each point with the integration domains and the two VDFs for the jet and plate surfaces [12].

(2) With the above question properly answered, can the above solutions to collisionless jet impingement loads offer bounding limits for less rarefied jets? This question seems simple, but must be addressed, which is the goal of this paper.

\subsection{Collisionless Jet Impingement Flow from a Finite Size Planar Exit}

Figure 1 illustrates the planar jet impingement flow problem. A jet fires from a planar exit, and impinges at an inclined plate. The jet number density, bulk velocity, and temperature are represented as $n_{0}, U_{0}$, and $T_{0}$. For convenience, the bulk velocity is further normalized as $S_{0}=U_{0} / \sqrt{2 R T_{0}}$, where $R$ is the gas constant. The plate inclination angle is denoted as $\alpha_{0}$, and the plate temperature is $T_{w}$. The exit semi-height is $H$, the plate semi-length is $W$, and the center-to-center distance from the exit to the plate is $L$. The plate surface can be either diffuse or specular.

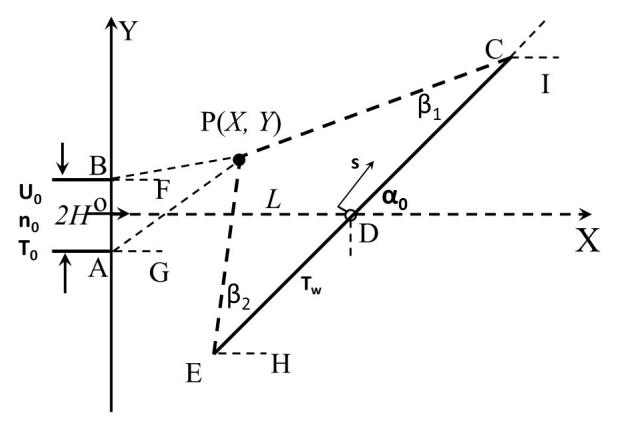

Figure 1. A free jet impinging at an inclined planar plate, $\angle P O X=\theta_{0}, \angle P B F=\theta_{1}, \angle P A G=\theta_{2}[19,20]$. (Reproduced from [19]. Copyright by the author, 2016.) 
Several related geometric expressions are defined and appear in the final expressions:

$$
\tan \theta=\frac{Y-y}{X}=\frac{v}{u+U_{0}},-H<y<H ; \tan \theta_{1}=\frac{Y-H}{X}, \tan \theta_{2}=\frac{Y+H}{X},
$$

where $(X, Y)$ is a point in the flowfield, it can be on the plate surface, and $(0, y)$ is a point at the nozzle exit.

The formulas for coefficients of local surface pressure, shear stress, and heat fluxes over a diffuse plate (i.e., $C_{p, d}(s), C_{f, d}(s)$, and $\left.C_{q, d}(s)\right)$ are derived with the gaskinetic theory, and the final expressions are [19]:

$$
\begin{gathered}
C_{p, d}(s)=\frac{2 e^{-S_{0}^{2}}}{\pi S_{0}^{2}} \int_{\theta_{1}}^{\theta_{2}} A_{1}(a) \sin ^{2}\left(\alpha_{0}-\theta\right) d \theta+\frac{\epsilon}{2 S_{0}^{2}} \frac{n_{w}(s)}{n_{0}}, \\
C_{f, d}(s)=\frac{e^{-S_{0}^{2}}}{\pi S_{0}^{2}} \int_{\theta_{1}}^{\theta_{2}} A_{1}(a) \sin \left(2 \alpha_{0}-2 \theta\right) d \theta, \\
C_{q, d}(s)=\frac{e^{-S_{0}^{2}}}{2 \pi S_{0}^{3}} \int_{\theta_{1}}^{\theta_{2}} A_{2}(a) \sin \left(\alpha_{0}-\theta\right) d \theta-\frac{n_{w}}{n_{0}} \frac{\epsilon^{3 / 2}}{\sqrt{\pi} S_{0}^{3}}, \\
A_{1}=\frac{\sqrt{\pi}}{4}[1+\operatorname{erf}(a)] e^{a^{2}}\left(3 a+2 a^{3}\right)+\frac{1}{2}+\frac{a^{2}}{2}, \epsilon=T_{w} / T_{0}, \\
A_{2}=\frac{\sqrt{\pi}}{2}\left[2+7 a^{2}+2 a^{4}\right] e^{a^{2}}[1+\operatorname{erf}(a)]+3 a+a^{3}
\end{gathered}
$$

where $a=S_{0} \cos \theta, \epsilon=T_{w} / T_{0}, n_{w}(s)$ is the number density for the Maxwellian VDF at the plate surface, and its value is determined by the non-penetration boundary conditions at the plate surface. The dynamic pressure $\rho_{0} U_{0}^{2} / 2$ at the nozzle exit is used to normalize the surface pressure and friction, and $\rho_{0} U_{0}^{3} / 2$ is used to normalize the heat flux at the plate surface, where $\rho_{0}$ is the bulk density at the jet exit. At the plate surface, pressure and heat flux are computed along the plate normal direction, while shear stress is computed along the direction parallel to the plate surface. A "virtual nozzle" treatment is adopted to investigate the specular reflective plate scenario [20], and it is quite successful. The pressure coefficient on a specular reflective plate surface is:

$$
C_{p, s}(s)=\frac{4 e^{-S_{0}^{2}}}{\pi S_{0}^{2}} \int_{\theta_{1}}^{\theta_{2}} A_{1}(a) \sin ^{2}\left(\alpha_{0}-\theta\right) d \theta .
$$

Shear stress and heat flux on a specular reflective plate are zero due to the symmetry condition [12]. More details about the treatments and results can be found in two recent papers $[19,20]$.

\subsection{Collisionless Jet Impingement Flow on a Rectangular Plate, from a Finite Size Round Exit}

Figure 2 illustrates the problem. The surface pressure coefficient at point $P(X, Y, Z)$ on the plate surface, or $P(s, \tau)$ in a local coordinate system on the plate surface, is:

$$
\begin{gathered}
C_{p, d}=\frac{2}{S_{0}^{2}} \frac{e^{-S_{0}^{2}}}{\sqrt{\pi}^{3} X^{2}} \int_{0}^{R_{0}} r d r \int_{0}^{2 \pi} \frac{A_{3}(a)}{Q^{5}}\left[\sin \alpha_{0}-B_{1} \cos \alpha_{0}\right]^{2} d \theta+\frac{\epsilon}{2 S_{0}^{2}} \frac{n_{w}(s, \tau)}{n_{0}} \\
A_{3}=\frac{\sqrt{\pi}}{4}[1+\operatorname{erf}(a)] e^{a^{2}}\left(2 a^{4}+6 a^{2}+\frac{3}{2}\right)+\frac{a^{3}}{2}+\frac{5}{4} a,
\end{gathered}
$$

where $R_{0}$ is the exit radius, and

$$
Q^{2}=\frac{X^{2}+Y^{2}+Z^{2}+r^{2}-2 r Y \cos \theta-2 Z r \sin \theta}{X^{2}}, B_{1}=(Z-r \sin \theta) / X
$$




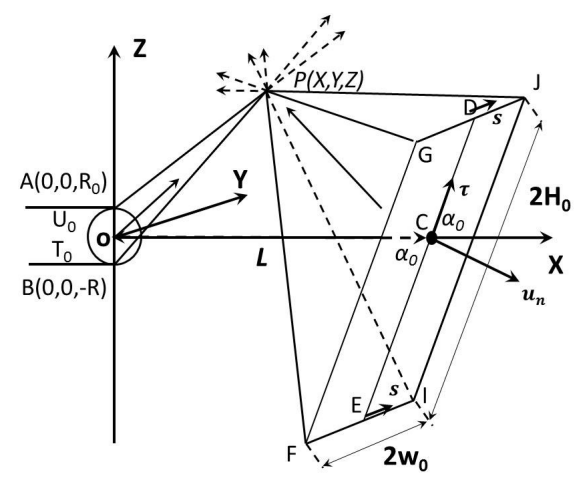

Figure 2. A free round jet impinging at an inclined planar plate.

For a diffuse flat surface, there are two friction forces along the plate surface. The friction coefficient related to the plate inclination direction (i.e., parallel to line $F G$ in Figure 2) is:

$$
C_{f, \tau}=\frac{2}{S_{0}^{2}} \frac{e^{-S_{0}^{2}}}{\sqrt{\pi} X^{2}} \int_{0}^{R_{0}} \frac{A_{3}(a)}{Q^{5}} r d r \int_{0}^{2 \pi}\left[\frac{1-B_{1}^{2}}{2} \sin 2 \alpha_{0}-B_{1} \cos 2 \alpha_{0}\right] d \theta
$$

The other friction force is along the direction parallel to line FI in Figure 2:

$$
C_{f, s}=\frac{2}{S_{0}^{2}} \frac{e^{-S_{0}^{2}}}{\sqrt{\pi}^{3} X^{2}} \int_{0}^{R_{0}} \frac{A_{3}(a) B_{2}}{Q^{5}} r d r \int_{0}^{2 \pi}\left(\sin \alpha_{0}-B_{1} \cos \alpha_{0}\right) d \theta
$$

The heat flux over a diffuse plate is along the plate normal direction, and the expression is:

$$
C_{q}=\frac{1}{\sqrt{\pi} S_{0}^{3}}\left(\frac{e^{-S_{0}^{2}}}{\pi X^{2}} \int_{0}^{R_{0}} \frac{A_{4}(a)}{Q^{4}}\left(\sin \alpha_{0}-B_{1} \cos \alpha_{0}\right) r d r \int_{0}^{2 \pi} d \theta-\sqrt{\epsilon^{3}} \frac{n_{w}(s, \tau)}{n_{0}}\right)
$$

where $A_{4}$ is a complex expression [19].

For a specular reflective surface, the shear stress and heat flux are zero, and the surface pressure coefficient is:

$$
C_{p, s}=\frac{4}{S_{0}^{2}} \frac{e^{-S_{0}^{2}}}{\sqrt{\pi^{3} X^{2}}} \int_{0}^{R_{0}} r d r \int_{0}^{2 \pi} \frac{A_{3}(a)}{Q^{5}}\left[\sin \alpha_{0}-B_{1} \cos \alpha_{0}\right]^{2} d \theta
$$

The above formulas for the local load are concise, with several parameters explicitly embedded. With a simple glance at these parameters by varying their values, the contribution to the results can be predicted. The embedded definite integrals in the above expressions can be conveniently evaluated with simple summations via a computer program. Once coded, predictions can be completed within seconds, and these formulas can significantly reduce time. By comparison, numerical simulations such as the Monte Carlo method may require days or weeks, and key physical insights are buried in large amounts of numerical output.

\section{Rarefication Effect on Local Surface Loads}

The above new formulas for the collisionless jet impingement surface loads were obtained and validated recently. It is natural to answer the second question mentioned in the previous section: how can we effectively use them for less rarefied jet impingement flows, and how confidently can we use these new formulas for impingement flows of low Kn numbers? To answer this question, two sets of DSMC simulations were performed. One set of simulations are for the planar exit scenario, and the other set of simulations are for the round exit scenario. The jet flows vary from continuum to collisionless, and the $K n$ numbers are $0.001,0.01,0.1,1,10,100$, and 10,000. The simulation domains and geometries are illustrated with Figures 1 and 2, and they can be found from two recent papers $[19,20]$. 
The time step and number of particles per cell in the final stage vary with different $K n$ numbers, and other major parameters are listed as follows.

Case I: Planar jet impingement at an inclined planar plate. The exit semi-height is set as $H=0.5 \mathrm{~m}$; the plate semi-height is set to $W=4 \mathrm{H}$; center-to-center distance from the nozzle exit to the plate is $L=4 \mathrm{H}$; the plate surface is set as diffuse and specular; the ratio between the plate temperature and exit gas is $T_{w} / T_{0}=1.5$; the exit speed ratio is $S_{0}=2.0$; and the plate inclination angle is $\alpha=60^{\circ}$. The plate surface is discretized with 200 points, and about 500,000 cells are used to discrete the two-dimensional simulation domain. In the final steady state, the numbers of particles vary from 0.2 million to 0.5 million, depending on different $K n$ numbers.

Case II: Round jet impingement at an inclined rectangular plate. The round exit radius is set as $D=0.5 \mathrm{~m}$; the plate width and height are set to $H=W=2 \mathrm{~m}$; the center-to-center distance from the nozzle exit to the plate is $L=2 \mathrm{~m}$; the plate surface is set as diffuse and specular; the ratio between the plate temperature and exit gas is $T_{w} / T_{0}=1.5$; the exit speed ratio is set as $T_{w} / T_{0}=1.5$, the exit speed ratio $S_{0}=2.0$, and the plate inclination angle is set as $\alpha=60^{\circ}$. The plate surface is triangulated with 2300 cells, and about 0.5 million tetrahedral cells are used to discrete the three-dimensional simulation domain. In the final steady state, the numbers of particles vary from 1.2 million to 3 million, depending on different $K n$ numbers. These three-dimensional simulations took a much longer time than the planar flow simulations in Case I.

A well-tested DSMC implementation package named GRASP [21] is adopted to perform the DSMC simulations. The hard sphere model is adopted for particle collisions, and standard DSMC sampling processes [12] are used to compute the pressure, shear stress, and heat flux over the plate surface.

Figures 3 and 4 show the pressure coefficients over a diffuse or a specular reflective surface, $C_{p, d}(K n)$ and $C_{p, s}(K n)$, for the planar exit scenario. Because the results are normalized, the results actually represent the averaged contributions to the plate per particle. One curve representing the analytical formulas at the collisionless flow limit is included in each figure (i.e., Equations (2) or (7)), and the parameters are listed above as Case I. DSMC simulation results with different $K n$ numbers are included in each figure. Several conclusions can be drawn from these two figures: (1) At the free molecular (FMF) or collisionless flow limit, the DSMC simulation results and the analytical expressions are essentially identical. (2) The pressure loads can be categorized into three groups: (i) Curves for $K n=10,000,100$, and 10 are much closer to the collisionless flow limits; (ii) $K n=0.1,0.01$, and 0.001, the jet flows are dense; (iii) Between these two groups is the curve for $K n=1$. (3) As the $K n$ number decreases, the peak pressure load decreases because more collisions scatter off high-energy particles; this is also illustrated by the increasing pressure loads at the two plate ends. (4) For this parameter combination, the diffuse plate has lower pressure load peaks at the collisionless flow limit, but the peak at the continuum flow regime is almost the same. These two results may not be generally correct. This is because the pressure loads on a specular plate do not include a temperature ratio, but the diffuse plate result does. At the collisionless limit, there are high-energy particles with very large velocities, and for less rarefied jets with smaller Kn numbers, there are more collisions which always reduce the number of particles with high velocity.

It is worth mentioning that one potential application for these analytical expressions is to use them as bounding curves to interpolate pressure coefficients for flows of finite Kn numbers. The other bounding curve is at the continuum flow limit, and it can be obtained by experiments or simulations. There may be many possible interpolation approaches; for example, for surface loads over a diffuse reflective plate at a finite $\mathrm{K} n$ number, the following formula may be feasible:

$$
C_{p, d}(K n)=A(1 / K n) C_{p, d}(K n=\infty)+B(K n) C_{p, d}(K n=0)
$$

where the term on the left side is the pressure load over a flat planar diffuse plate; $C_{p, d}(K n=\infty, s)$ is the surface pressure load at the collisionless flow limit (Equation (2)); $C_{p, d}(K n=0, s)$ is the surface loads at the continuum flow limit; $A(1 / K n)$ and $B(K n)$ are two weighting factors. In Figure 3 , an interpolation 
curve of $K n=1$ is included by simply averaging the simulation results of flows with $K n=0.001$ and $K n=\infty$. As is shown, the interpolated results are actually very close to the DSMC simulation results for $K n=1$.

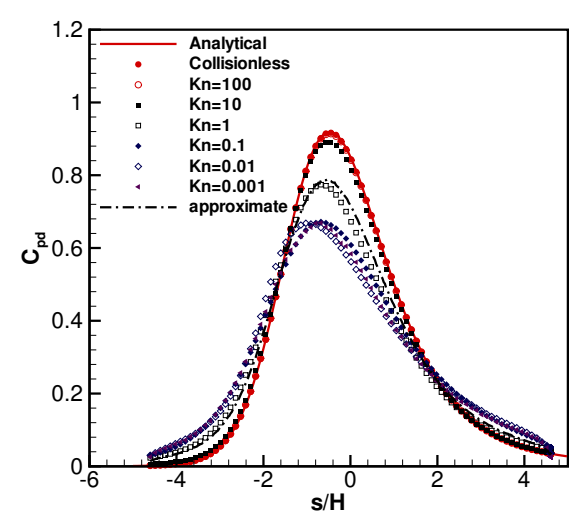

Figure 3. Rarefication effects on $C_{p, d}(s)$, diffuse plate, and from a planar exit. Lines: analytical; Symbols: direct simulation Monte Carlo (DSMC). $T_{w} / T_{0}=1.5, L /(2 H)=4.0, \alpha_{0}=60^{\circ}$, and $S_{0}=2.0$.

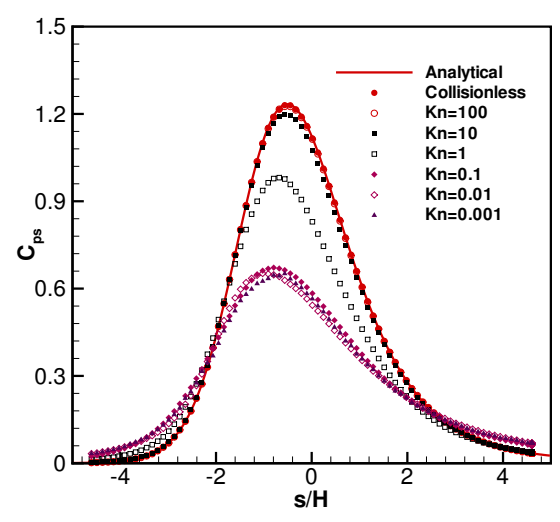

Figure 4. Rarefication effect on $C_{p, s}(s)$, specular plate, and from a planar exit. Lines: analytical; Symbols: DSMC. $T_{w} / T_{0}=1.5, L /(2 H)=4.0, \alpha_{0}=60^{\circ}$, and $S_{0}=2.0$.

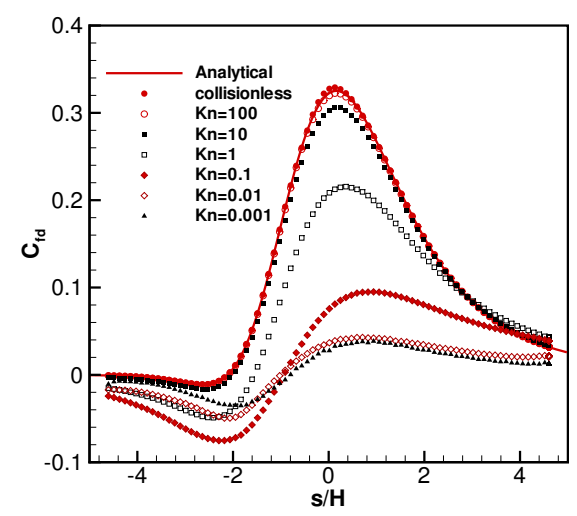

Figure 5. Rarefication effects on $C_{f, d}(s)$, diffuse plate, and from a planar exit. Lines: analytical; Symbols: DSMC. $T_{w} / T_{0}=1.5, L /(2 H)=4.0, \alpha_{0}=60^{\circ}$, and $S_{0}=2.0$. 
Figures 5 and 6 are the corresponding coefficients for local friction and heat loads on a diffuse reflective plate. Each figure includes one curve calculated with Equations (3) and (4), for flows at the collisionless limit. DSMC simulation results are also included. These two figures indicate that it is rather difficult to predict the $K n$ number effect; even though the peak friction values continue to decrease around $s / H=0.5$, local friction coefficient may increase or decrease, around $s / H=3.0$. Figure 6 indicates that the heat flux coefficient profiles continue to decrease with denser jet flows. The DSMC simulation results can be categorized into three groups as well. However, different from the pressure loads (which are always positive), the friction force can be positive or negative; i.e., along two directions parallel to the plate surface. The heat flux can be from a hot plate to a cold jet flow, or from a hot jet flow to a cold plate; hence, the flux can be either positive or negative as well.

Three-dimensional DSMC simulations are also performed for the scenario of a round jet impinging at a rectangular flat plate; the parameters are listed above as Case II.

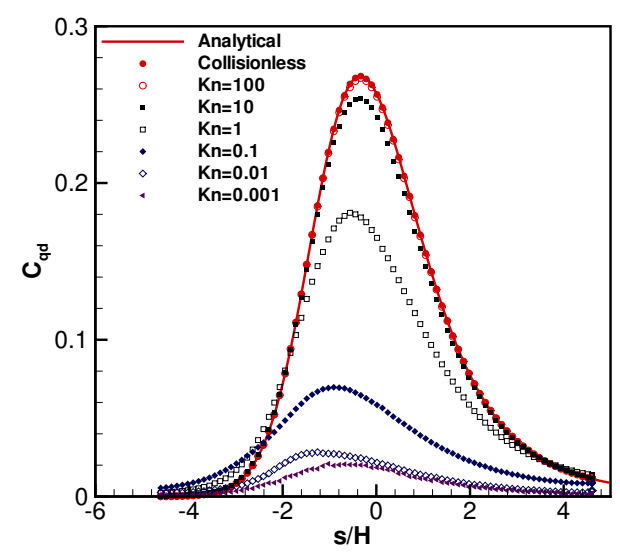

Figure 6. Rarefication effects on $C_{q, d}(s)$, diffuse reflective plate, from a planar exit. Lines: analytical; Symbols: DSMC. $\alpha_{0}=60^{\circ}, S_{0}=2.0, T_{w} / T_{0}=1.5$, and $L /(2 H)=4.0$.

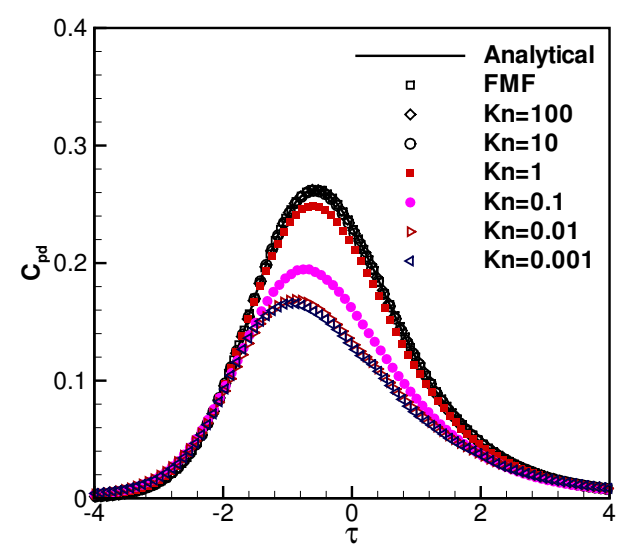

Figure 7. Rarefication effects on surface pressure, $C_{p, d}$, along a diffuse plate centerline. Lengths are normalized by the round nozzle diameter. Solid: analytical; Dashed: DSMC. $S_{0}=2.0, T_{w} / T_{0}=1.5$, $\alpha_{0}=60^{\circ}, L / D=4$. FMF: free molecular flow.

Figures 7 and 8 show the pressure loads along the rectangular plate centerline $D E$ in Figure 2. As expected, similar trends are observed here, corresponding to the planar flow case. For the free molecular flow situation, the simulation and analytical results are essentially identical, indicating that for this three-dimensional flow situation, Equations (8) and (14) for the pressure loads at the 
collisionless flow limit are accurate. The pressure peaks are much higher than less rarefied jet flow situation. For this set of parameters, the peak value of $C_{p, s}$ is much larger than $C_{p, d}$.

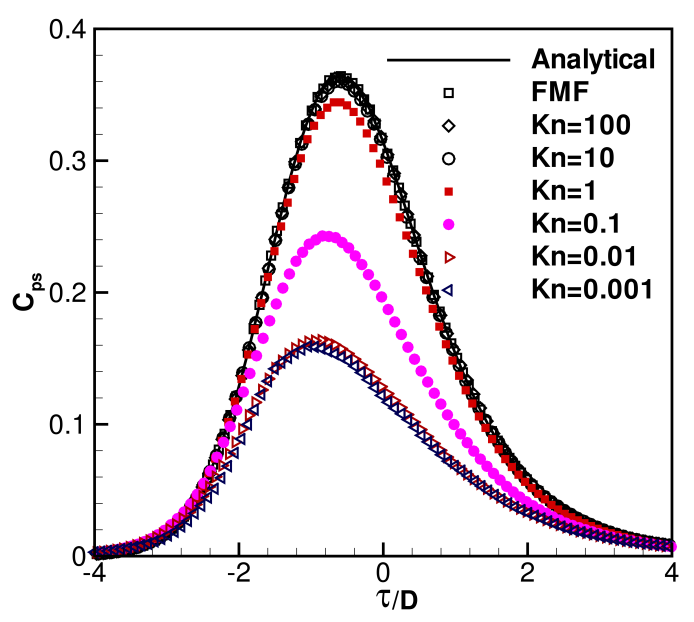

Figure 8. Rarefication effects on surface pressure, $C_{p, s}$, along a specular plate centerline. Lengths are normalized by the nozzle diameter. Solid: analytical; Dashed: DSMC. $S_{0}=2.0, T_{w} / T_{0}=1.5, \alpha_{0}=60^{\circ}$, $L / D=4$.

Figures 9 and 10 are the corresponding surface friction and heat flux distributions along the same rectangular plate centerline. At the collisionless flow limit, the simulation results and the analytical formulas, Equations (12) and (13) are identical. In general, as the flow becomes less rarefied, the peak values for friction and heat load decrease, and the curves change mildly. However, there are two local peaks in the friction distribution profiles, and the smaller one does not change monotonically. This is understandable, because collisions scatter off molecules and even change their directions; hence, the flowfield patterns may change.

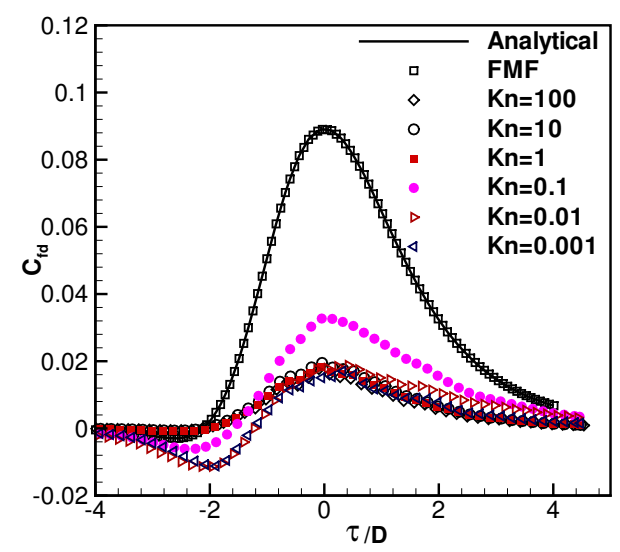

Figure 9. Rarefication effects on plate surface friction, $C_{f 1, d}$, along a diffuse plate centerline. Lengths are normalized by the nozzle diameter. Solid: analytical; Dashed: DSMC. $S_{0}=2.0, T_{w} / T_{0}=1.5$, $\alpha_{0}=60^{\circ}, L / D=4$.

For this three-dimensional flow scenario, molecules have larger freedom to change flow directions than a planar exit flow situation, and they scatter off more effectively. For the heat flux loads, Figure 10 indicates that there are three groups of curves with this set of parameter combinations: $K n>1, K n=1$, and $K n<1$. 


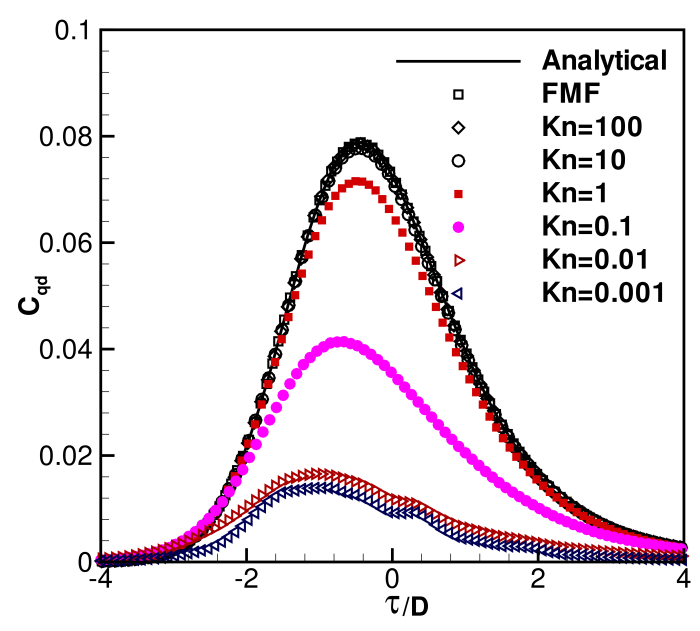

Figure 10. Rarefication effects on plate surface heat flux, $C_{q, d}$, along a diffuse plate centerline. Lengths are normalized by the nozzle diameter. Solid: analytical; Dashed: DSMC. $S_{0}=2.0, T_{w} / T_{0}=1.5$, $\alpha_{0}=60^{\circ}$, and $L / D=4$.

The surface pressure coefficients are always positive, and those inter-molecule collisions tend to create a relatively uniform pressure distribution along the surface. For the surface friction forces and heat fluxes, they can be either positive or negative, depending on the flow directions along the surface and the flow and wall temperatures. It is improper to declare that less rarefied flows tend to create more uniform surface friction and heat load distributions, but it is fair to draw the conclusion that the concise expressions for the friction and heat loads at the collisionless flow limit may offer reasonable references.

\section{Conclusions}

Rarefication effects on jet impingement loads at a flat plate are investigated. DSMC simulations are performed, and the results are compared with analytical expressions for surface loads at the collisionless flow limit. In these DSMC simulations, the jet flows have different $K n$ numbers, and they can be continuum, near continuum, transitional, or collisionless.

The purpose of this paper is to answer the question of how confidently we can be in using the new surface load formulas obtained recently $[19,20]$ for less-rarefied jet flows. These results indicate that the analytical formulas for surface loads provide upper limits for the largest load peaks; hence, these formulas at the collisionless flow limit can offer quick references for the less-rarefied flow scenario. In general, in flows with higher densities and more collisions, the profiles level off and spread out. However, it is improper to declare that local properties must decrease monotonically; for example, the surface friction and heat flux coefficients may have different local variations. These results can help applications in space engineering and can reduce the amount of simulations and experiments. Based on these expressions for local surface loads, it is further possible to obtain expressions for the total surface loads at the collisionless flow limit [20]. It is reasonable to believe that those global load coefficients can also be used as references for less-rarefied jet impingement loads at a plate.

Author Contributions: Shiying Cai performed numerical simulations; Kai Zhang verified all equations; Chunpei Cai and Jun Li wrote the paper.

Conflicts of Interest: The authors declare no conflict of interest. 


\section{References}

1. Maev, R.; Leshchynsky, V. Introduction to Low Pressure Gas Dynamic Spray; Wiley-Vch: Weinheim, Germany, 2008.

2. Sanna, G.; Tomassetti, G. Introduction to Molecular Beams Gas Dynamics; Imperial College Press: London, UK, 2005.

3. Metzger, P. Rocket exhaust cratering: A serious challenge for space exploration. In Proceedings of the 1st Workshop on Granular Materials in Lunar and Martian Exploration, Cocoa Beach, FL, USA, 3-4 February 2005.

4. Clark, L.V.; Land, N.S. Experimental Investigation of Jet Impingement on Surfaces of Fine Particles in a Vacuum Environment; National Aeronautics and Space Administration: Washington, DC, USA, 1965.

5. Hasting, D.; Garrett, H. Spacecraft-Environment Interactions; Cambridge University Press: Cambridge, UK, 1996.

6. Rebrov, A.K. Free jets in vacuum technologies. J. Vac. Sci. Techol. A 2001, 19, 1679-1687, doi:10.1116/1.1382649.

7. Liepmann, H.W. Gas-kinetics and gasdynamics of orifice flow. J. Fluid Mech. 1961, 10, 65-79, doi:10.1017/S002211206100007X.

8. Narasimha, R. Collisionless expansion of gases into vacuum. J. Fluid Mech. 1962, 12, 294-308, doi:10.1017/S0022112062000208.

9. Naumann, K.W. The freezing of flow deflection in Prandtl-Meyer expansion to vacuum. In Proceedings of the 15th International Symposium on Rarefied Gas Dynamics, Grado, Italy, 16-20 June 1986; Volume 2, pp. 524-533.

10. Naumann, K.W. Analytical method for rapid estimation of plumes from small satellite, attitude control thrusters. In Proceedings of the 17th International Rarefied Gas Dynamics Symposium, Aachen, Germany, 8-14 July 1990; VCH-Verlagsgesellschaft mbH: Weinheim, Germany; New York, NY, USA, 1991; pp. 971-978.

11. Simons, G.A. Effects of nozzle boundary layers on rocket exhaust plumes. AIAA J. 1972, 10, 1534-1535.

12. Bird, G.A. Molecular Gas Dynamics and The Direct Simulation of Gas Flows, 2nd ed.; Claredon Press: Oxford, UK, 1994.

13. Vashchenov, P.; Kudryavstev, A.; Khotyanovsky, D.; Ivanov, M. DSMC and Navier-Stokes study of backflow for nozzle plumes expanding into vacuum. In Proceedings of the 24th International Symposium on Rarefied Gas Dynamics, Monopoli, Italy, 10-16 July 2004; AIP Conference Proceedings: Melville, NY, USA; Volume 762, pp. 355-360.

14. Kannenberg, K.C.; Boyd, I.D. Three-dimensional Monte Carlo simulation of plume impingement. J. Thermophys. Heat Transf. 1999, 13, 226-235, doi:10.2514/2.6440.

15. Noller, H.G. Approximate calculation of expansion of gas from nozzles into high vacuum. J. Vac. Sci. Techol. 1966, 3, 202-207, doi:10.1116/1.1492475.

16. Woronowicz, M.S.; Rault, N. On jet flowfield analysis and simulation techniques. In Proceedings of the 6th AIAA/ASME Joint Thermophysics and Heat Transfer Conference, Colorado Springs, CO, USA, 20-23 June 1994; AIAA Paper 1994-2048.

17. Chen, $X$. The impact force acting on a flat plate exposed normally to a rarefied plasma plume issuing from an annular or circular nozzle. J. Phys. D 2010, 43, 315205, doi:10.1088/0022-3727/43/31/315205

18. Dettleef, G. Plume flow and plume impingement in space technology. Prog. Aerosp. Sci. 1991, 28, 1-71, doi:10.1016/0376-0421(91)90008-R.

19. Cai, C. Gaskinetic modeling on dilute gaseous plume impingement flows. Aerospace 2016, 3, 43, doi:10.3390/aerospace3040043.

20. Cai, C.; He, X. Detailed flow field and surface properties for high Knudsen number planar jet impingement at an inclined flat plate. Phys. Fluids 2016, 28, 056103, doi:10.1063/1.4948365.

21. Liu, H.; Cai, C.; Zou, C. An object-oriented implementation of the DSMC method. Comput. Fluids 2012, $57,65-75$.

(C) 2017 by the authors. Licensee MDPI, Basel, Switzerland. This article is an open access article distributed under the terms and conditions of the Creative Commons Attribution (CC BY) license (http://creativecommons.org/licenses/by/4.0/). 